\title{
EVALUACIÓN DE LA COMPACTACIÓN Y CBR DE UN MATERIAL TIPO SUBBASE CON ADICIÓN DE CENIZA DE BAGAZO DE CAÑA DE AZÚCAR
}

\author{
D. L. GARCÍA-GARCÍA ${ }^{1}$, J. L. PÉREZ-DÍAZ² ${ }^{2}$ C. GAONA-TIBURCIO ${ }^{3}$, \\ F. ALMERAYA-CALDERÓN ${ }^{3}$, J. M. MENDOZA-RANGEL ${ }^{4}$, A. LANDA-GÓMEZ ${ }^{5}$, \\ O. M. LÓPEZ-YZA ${ }^{1}$, S. MÁRQUEZ MONTERO ${ }^{1}$, M. A. BALTAZAR-ZAMORA ${ }^{1 *}$ \\ ${ }^{1}$ Universidad Veracruzana, Facultad de Ingeniería Civil-Xalapa, Circuito G. Aguirre Beltrán S/N, \\ Lomas del Estadio, Xalapa, Veracruz, México, C.P 91000 \\ ${ }^{2}$ Universidad Autónoma de Chiapas, Facultad de Ingeniería, Tuxtla Gutiérrez, Chiapas, México \\ ${ }^{3}$ Universidad Autónoma de Nuevo León, FIME - CIIIA., Av. Universidad S/N, Ciudad Universitaria, \\ San Nicolás de los Garza, N.L., México, CP 66451 \\ ${ }^{4}$ Universidad Autónoma de Nuevo León, FIC, San Nicolás de los Garza, N.L. México. \\ ${ }^{5}$ Universidad Veracruzana, Centro de investigación en Micro y Nanotecnología, Boca del Río, Veracruz, México.
}

\section{RESUMEN}

En el presente trabajo de investigación se empleó la ceniza de bagazo de caña de azúcar (CBCA) como sustituto parcial al cemento portland con la finalidad de mejorar las propiedades mecánicas del suelo de estudio. Se evaluaron los pesos volumétricos secos máximos, humedad optima, compactación y CBR, de un suelo tipo Subbase comparándolo con mezclas de suelo con adiciones del 3\%, 5\% y $7 \%$ de CPC, CBCA y combinaciones de CPC-CBCA. Todos los ensayos se realizaron de acuerdo con la normativa de la SCT para el control de materiales y ensayos mencionados. Los resultados mostraron mejoras significativas en el CBR, con sustitución de hasta $25 \%$ y hasta 50\% de CBCA por CPC.

Palabras clave: Ceniza de bagazo de caña de azúcar

\begin{abstract}
In the present research work sugar cane bagasse ash (CBCA) was used as a partial substitute to Portland cement in order to improve the mechanical properties of the study soil. The maximum dry volumetric weights, optimum humidity, compaction and CBR, of a Sub-base soil type were evaluated, comparing it with soil mixtures with additions of $3 \%, 5 \%$ and $7 \%$ of CPC, CBCA and CPC-CBCA combinations. All the tests were carried out in accordance with the regulations of the SCT for the control of materials and tests mentioned. The results showed significant improvements in the CBR, with substitution of up to $25 \%$ and up to $50 \%$ of CBCA by CPC.
\end{abstract}

Keywords: Sugarcane Bagasse Ash.

\section{RESUMO}

No presente trabalho de pesquisa, o bagaço de cana-de-açúcar (CBCA) foi utilizado como substituto parcial do cimento Portland, a fim de melhorar as propriedades mecânicas do solo estudado. Avaliaram-se os pesos volumétricos secos máximos, umidade ótima, compactação e CBR, de um tipo de solo Sub-base, comparando-os com misturas de solo com adições de 3\%, 5\% e 7\% das combinações CPC, CBCA e CPC-CBCA. Todos os testes foram realizados de acordo com os regulamentos da SCT para o controle de materiais e testes mencionados. Os resultados mostraram melhorias significativas no CBR, com substituição de até $25 \%$ e até $50 \%$ do CBCA pelo CPC.

Palavras chave: Cinza de bagaço de cana 


\section{INTRODUCCIÓN}

El suelo es uno de los materiales más importante en la construcción de obras de infraestructura vial, por lo que debe de tenerse un estricto control de calidad para que pueda desempeñar una vida de servicio adecuada. Sin embargo, en muchas ocasiones el suelo carece de las características necesarias para su empleo en la construcción, por lo cual se debe de aplicar en ellos un procedimiento de mejoramiento con el fin de obtener mejores características físicas, mecánicas y químicas (Fernández Loaiza, 1982). Entre los procedimientos más empleados se encuentra la adición de agentes físicos-químicos, como son el uso de cemento portland, cal, asfalto, ceniza volante, humo de sílice, escoria de alto horno, entre otros materiales alternativos. Actualmente la Ceniza de Bagazo de Caña de Azúcar se encuentra en constante investigación debido a sus propiedades puzolánicas y a la gran producción de este producto agroindustrial en el mundo (Ariza H. et.al., 2019; Baltazar M. et.al., 2016; Cordeiro G. et.al., 2008; García D. et.al., 2019; Landa A. et.al.,2019; Landa A.E. et.al., 2018; Ruíz, M. 2019; Santiago, G.2016). Empleando principalmente la CBCA como en reemplazo parcial del CP para la elaboración de concretos y morteros, representando un mejoramiento físico y químico del suelo y una disminución en la contaminación por la industria del cemento (Moraes et al., 2015).

El presente trabajo de investigación tiene como objetivo determinar la influencia de la sustitución parcial del CP por CBCA en las propiedades mecánicas de un suelo empleado como subbase, realizando ensayes de compactación, contenido de húmedas, límites de consistencia de Atterberg, CBR, expansión y resistencia a la compresión simple. Con la finalidad de generar una disminución de $\mathrm{CO}_{2}$ y obtener mejoras en las propiedades del suelo. Se diseñaron doce tipos de mezclas con porcentajes de sustitución del 3\%, 5\% y 7\% respecto a la masa y con combinaciones de CP y CBCA de $100 \%, 75 \%, 50 \%$ y $0 \%$.

\section{METODOLOGÍA EXPERIMENTAL}

La metodología empleada en esta investigación se encuentra dividida en dos secciones principales las cuales comprenden las características fisicoquímicas de la CBCA y los ensayes mecánicos que se realizaron al suelo de estudio con las diferentes combinaciones de sustitución parcial.

\subsection{Materiales de estudio}

Los materiales de estudio que se seleccionaron fueron, una muestra representativa de un material de subbase adquirido del banco de materiales el palmar en la carretera federal Xalapa-Veracruz, México. El muestreo, identificación, preparación de la muestra y ensayos de calidad fueron efectuados de acuerdo con la normativa ONNCCE y ASTM para suelos.

Se utilizo Cemento Portland Compuesto, clase R-30 de alta resistencia de acuerdo con la norma mexicana NMX-C-414-ONNCCE (ONNCCE, 2014) y Ceniza de Bagazo de Caña de Azúcar proveniente del ingenio Mahuixtlan.

\subsection{Métodos de clasificación}

\subsubsection{Caracterización de materiales}

Se realizó la caracterización química de la CBCA mediante el análisis de fluorescencia de rayos X y estudios mineralógicos mediante difracción de rayos X. Mientras que al suelo de subbase se le realizaron parámetros geotécnicos como densidad, granulometría, límites de consistencia, compactación, CBR y expansión.

\subsubsection{Diseño de las mezclas de suelo}

El diseño de las mezclas se estableció con tres porcentajes de adición de CPC de acuerdo con la clasificación del material, tal como lo establecen diversos autores de la literatura (Jofre et al., 2008; Juarez Gutierrez \& Inzunza Ortiz, 2011). Los porcentajes establecidos fueron 3\%, 5\% y 7\% con 
respecto a la masa total del suelo seco. Se establecieron también, sustituciones parciales de CPC por CBCA en cada porcentaje anterior, de $0 \%, 25 \%, 50 \%$ y $100 \%$, teniéndose un total de 12 mezclas, como se muestra en la Tabla 1.

Tabla 1. Dosificación de adiciones en el suelo

\begin{tabular}{cccc}
\hline Suelo & Porcentaje de sustitución & CPC & CBCA \\
\hline 1 & $3 \%$ & $100 \%$ & $0 \%$ \\
2 & $5 \%$ & & \\
3 & $7 \%$ & $75 \%$ & $25 \%$ \\
4 & $3 \%$ & & \\
5 & $5 \%$ & & \\
6 & $7 \%$ & $50 \%$ & \\
7 & $3 \%$ & & \\
8 & $5 \%$ & & $100 \%$ \\
9 & $7 \%$ & $0 \%$ & \\
10 & $3 \%$ & & \\
11 & $5 \%$ & & \\
12 & $7 \%$ & & \\
\hline
\end{tabular}

\subsubsection{Pruebas realizadas al suelo con adiciones}

Se realizaron las siguientes pruebas a los doce tipos de suelo de estudio de acuerdo con la normativa, para evaluar los diferentes resultados.

\subsubsection{Compactación}

Se determino en cada tipo de suelo la masa volumétrica seca máxima y su contenido de agua óptimo, con la finalidad de analizar las variaciones en cada porcentaje de adición suelo-CPC, suelo CBCA y suelo con combinaciones CPC-CBCA y como dato para la posterior elaboración de especímenes.

Las pruebas se realizaron de acuerdo con la normativa SCT, variable D, la cual tiene como parámetros una muestra representativa de $7 \mathrm{~kg}$. Un molde de $152.4 \mathrm{~mm}$ de diámetro, una compactación dinámica con un pisón de $4.54 \mathrm{~kg}$. de peso, una altura de caída de $45.7 \mathrm{~cm}$ y cinco capas.

\subsubsection{Ensayo CBR y expansión}

Se realizó la prueba de valor soporte de california y expansión por saturación de los especímenes de estudios, para ello fueron compactados dinámicamente tres especímenes con diferentes energías de compactación y contenido óptimo de agua y saturados durante 24 horas simulando condiciones críticas, para obtener cambios volumétricos, para posteriormente ensayarlos en la prensa de carga. De acuerdo con la normativa SCT, M-MMP-1-1/16.

\section{ANÁLISIS Y RESULTADOS}

\subsection{Caracterización fisicoquímica de los materiales de estudio}

Los resultados de caracterización del suelo de estudio se presentan en la tabla 2, en ella se muestras todos los parámetros geotécnicos necesarios de cada material a emplear en la construcción de una infraestructura vial, mostrando que el suelo con base a los resultados granulométricos obtenidos y a los límites de consistencia se clasifica como una grava bien graduada con finos arcillosos no plásticos, con un $54 \%$ de gravas. En cuanto a las características de compactación se tiene que la humedad optima es de $9.1 \%$ con un peso volumétrico seco máximo de $2030 \mathrm{~kg} / \mathrm{m}^{3}$, y que con ese peso volumétrico los valores de soporte de california se encuentran en $102.9 \%$ con $0 \%$ de expansión, cumpliendo con las características para ser empleado como materia de subbase, como lo establecen las normas mexicanas. 
Tabla 2. Propiedades geotécnicas del suelo

\begin{tabular}{cc}
\hline Propiedades del suelo & Resultados \\
\hline Humedad natural (\%) & N/A \\
Pasa la malla 3” (\%) & 100 \\
Pasa la malla N. 4 (\%) & 46 \\
Pasa la malla N. 200 (\%) & 10 \\
Límite líquido (\%) & 26 \\
Límite plástico (\%) & N/A \\
Índice plástico (\%) & N/A \\
Peso volumétrico seco máximo $\left(\mathrm{kg} / \mathrm{m}^{3}\right)$ & 2030 \\
Humedad óptima (\%) & 9.1 \\
Valor Relativo de Soporte $(\%)$ & 102.9 \\
Expansión (\%) & 0 \\
\hline
\end{tabular}

Mientras que la Tabla 3 presenta los resultados de la composición química de la CBCA, donde se puede observar que la suma de los óxidos principales $\mathrm{SiO}_{2}+\mathrm{Al}_{2} \mathrm{O}_{3}+\mathrm{Fe}_{2} \mathrm{O}_{3}$ representa un total de $73.069 \%$ encontrándose por encima del 70\% que establece la norma ASTM C618 para puzolanas clase N y F. siendo estos resultados consistentes con los obtenidos por diversos autores que han reportados la composición química de la CBCA en diferentes lugares (Singh N.B. et al. 2000; Frías M.,2011).

Tabla 3. Composición química de la CBCA

\begin{tabular}{cc}
\hline Componente químico & Concentración \\
\hline $\mathrm{SiO}_{2}$ & 62.667 \\
$\mathrm{Al}_{2} \mathrm{O}_{3}$ & 5.208 \\
$\mathrm{Fe}_{2} \mathrm{O}_{3}$ & 5.194 \\
$\mathrm{TiO}_{2}$ & 0.647 \\
$\mathrm{CaO}$ & 4.875 \\
$\mathrm{MgO}$ & 2.194 \\
$\mathrm{SO}_{3}$ & 0.309 \\
$\mathrm{~K}_{2} \mathrm{O}$ & 13.93 \\
$\mathrm{Na}_{2} \mathrm{O}$ & 0.569 \\
$\mathrm{P}_{2} \mathrm{O}_{5}$ & 3.361 \\
\hline
\end{tabular}

El modelo de difracción se observa presencia de partículas amorfas en el halo localizado en la región $2 \theta$ de $10-40^{\circ}$, también se observa que la CBCA está compuesta principalmente por timidita el cual es un polimorfo del $\mathrm{SiO}_{2}$, cianita mineral de la familia de los silicatos, óxido de potasio, óxido de hierro y oxido de silicio y aluminio, encontrándose como materiales cristalinos. Difractogramas similares han sido obtenidos por diversos autores, coincidiendo en señalar la región anteriormente descrita (Jagadesh, P. et al. 2015; Torres B.J. et al. 2014) 


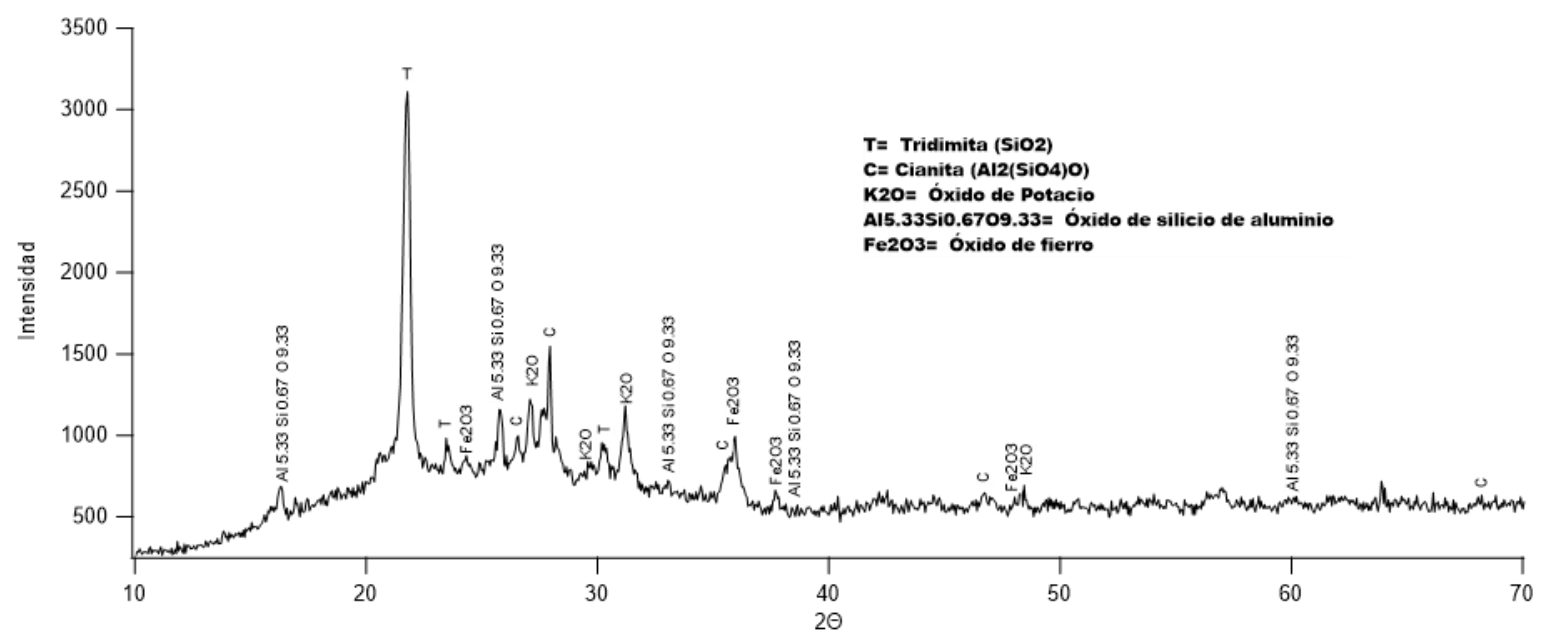

Figura 1. Modelo DRX de la CBCA

\subsection{Compactación del suelo}

En la figura 2 se observa la relación entre la masa volumétrica seca máxima del suelo respecto a el porcentaje de adición del estabilizante en diferentes porcentajes de CPC y CBCA. Teniendo el suelo en su estado natural una MVSM de $2030 \mathrm{~kg} / \mathrm{m}^{3}$, mostrando un resultado con adiciones del $100 \%$ CPC sin variaciones significativas al igual que las mezclas con el $100 \% \mathrm{CBCA}$ y la 50\%CPC y CBCA, a lo contrario de las adiciones con $75 \% \mathrm{CPC}$ y $25 \%$ CBCA las cuales incrementan la masa volumétrica seca máxima como consecuencia de una mejor densificación y distribución uniforme de las partículas de suelo, CPC y CBCA durante el proceso de compactación, logrando sellar los vacíos entre las partículas comprimidas, dado que los porcentajes de estabilizante van aumentado.

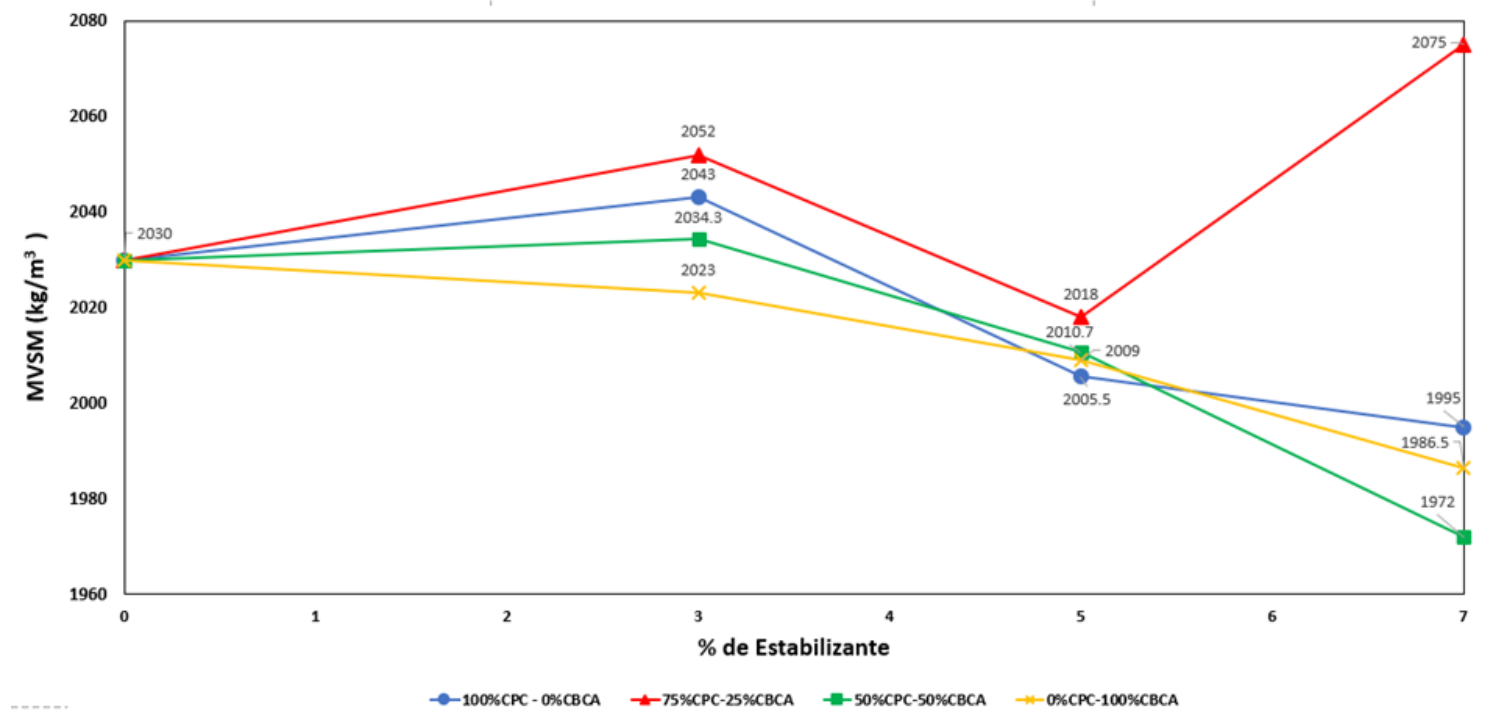

Figura 2 Relación de la MVSM y \% de estabilizante

Respecto a la humedad óptima necesaria para cada tipo de mezcla en la prueba de compactación muestra que respecto a la cantidad de adiciones disminuye o aumenta el agua, mostrando que a mayor cantidad de CBCA incrementa la absorción de agua por sus partículas, teniendo el mayor incremento de agua en la mezcla con el $7 \%$ de estabilizante de $100 \%$ CBCA, la cual no fue beneficio debido a que su masa volumétrica seca máxima es inferior al estado natural del suelo. Mientras que las mezclas de suelo con adiciones de $100 \%$ CPC muestran inicialmente una disminución de humedad, conforme se incrementa la cantidad de CPC se requiere una mayor cantidad de agua, como se muestra en la figura 3. 


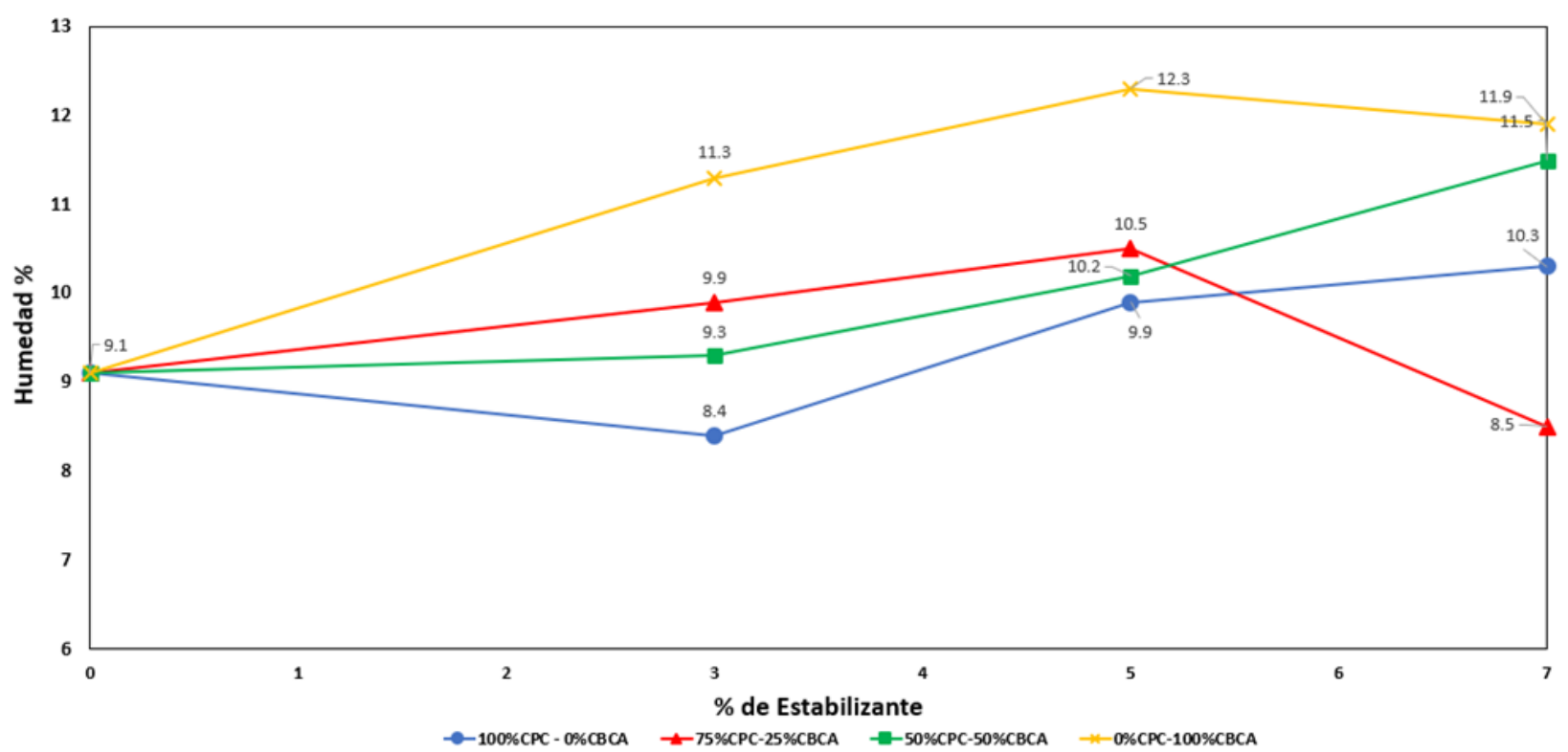

Figura 3. Relación de la humedad óptima y porcentaje de estabilizante

\subsection{CBR del suelo}

En la figura 4 se muestra el comportamiento de CBR de las distintas mezclas de suelo ensayadas, mostrando una mayor resistencia al adicionarle únicamente CPC, logrando obtener valores hasta del $159 \%$ en el $7 \%$ de estabilizante, mientras que al sustituir parcial o totalmente el porcentaje decrece, siendo el $100 \%$ de CBCA la mezcla con valores inferiores al estado natural de suelo del CBR. La mezcla con $75 \%$ de CPC y $25 \%$ CBCA presenta valores superiores al estado natural del suelo, teniendo un comportamiento creciente lineal, al contrario de las otras mezclas.

En general se observa un incremento en el valor de CBR con respecto al valor del suelo sin adiciones, ya que adicionar CPC únicamente, y teniendo un comportamiento similar las sustituciones de $25 \%$, logra que este posea significativos incrementos de reacciones puzolánicas entre la sílice amorfa de la ceniza y los iones de calcio del cemento portland. Mientras que el efecto de la finura de la ceniza de bagazo de caña de azúcar provoca una reacción opuesta, ya que al incrementar más su cantidad vuelve al suelo inestable y físicamente lodoso.

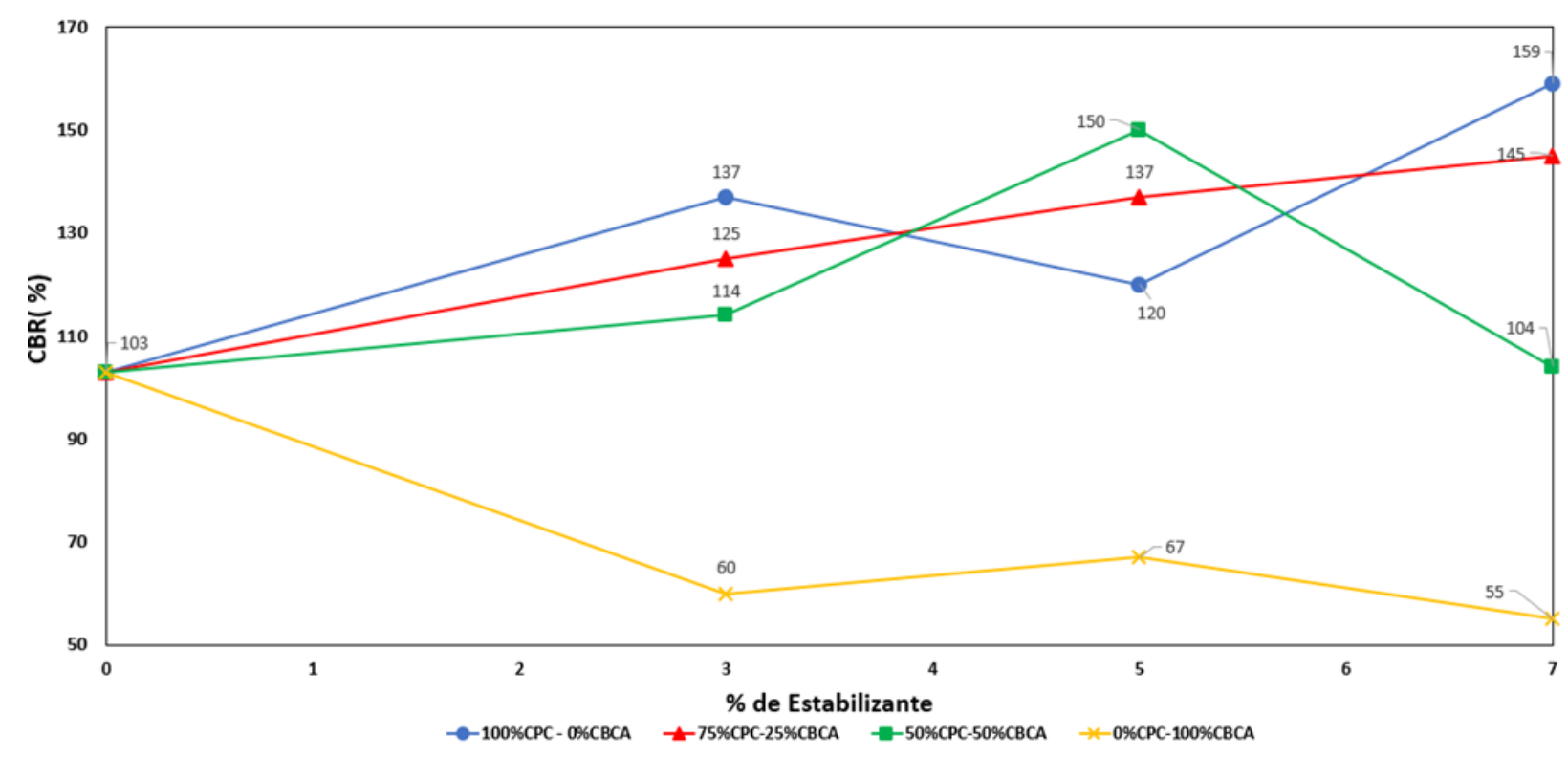

Figura 4 Relación de CBR y porcentaje de estabilizante 


\section{CONCLUSIONES}

Con base a resultados obtenidos podemos concluir que la mezcla referente al $100 \%$ de CPC presenta resultados con características favorables, seguido de la mezcla con el $75 \%$ CPC - 25\% CBCA, mostrando que las dos mezclas restantes al tener un alto contenido de CBCA disminuyen las propiedades mecánicas del suelo siendo no recomendables para el mejoramiento del suelo de sub-base, por lo cual se concluye que el uso de la CBCA pose resultados favorables cuando se emplea en un $25 \%$.

\section{AGRADECIMIENTOS}

M.A. Baltazar-Zamora, et. al., agradecen a PRODEP por el Apoyo otorgado por la SEP, al Cuerpo Académico UV-CA-458 "Sustentabilidad y Durabilidad de Materiales para la Infraestructura Civil", en el marco de la Convocatoria 2018 para Fortalecimiento de Cuerpos Académicos con IDCA 28593.

\section{REFERENCIAS}

Ariza H.A., Croche R., Gaona C., Almeraya F., Ramírez A.C., Villegas R., Márquez S., BaltazarZamora M.A. (2019). "Evaluación de la corrosión del acero galvanizado y acero AISI 304 embebidos enconcretos ecológicos con sustitución de CPC por 10, 20 y 30\% de CBCA-HS expuestos a Na2SO4 al 3.5\%". Memorias del XXXIV Congreso Nacional de la Sociedad Mexicana de Electroquímica, 12th Meeting of the Mexican Section of the Electrochemical Society, SMEQ, Querétaro (México).

Baltazar-Zamora M.A., Santiago, G., Moreno V., Croche R., De la Garza M., Estupiñan, F., Zambrano, P., Gaona, C. (2016). Electrochemical Behaviour of Galvanized Steel Embedded in Concrete Exposed to Sand Contaminated with $\mathrm{NaCl}$. Int. J. Electrochem. Sci. 11, 10306-10319, doi: https://doi.org/10.20964/2016.12.28.

Cordeiro, G.C., Toledo, R.D., Tavares, E.R., Fairbairn E.M. (2008). Pozzolanic activity and filler effect of sugar cane bagasse ash in Portland cement and lime mortars. Cement and Concrete Composites.30(5):410-418

Fernandez Loaiza, C. F. (1982). Mejoramiento y estabilización de suelos. D.F: LIMUSA.

Frias, M., Villar, E., Savastano, H. (2011). Brazilian sugar cane bagasse ashes from the cogeneration industry as active pozzolans for cement manufacture. Cement and Concrete Composites , 490-499.

García D.L, Cabral J.A, Gaona C., Almeraya F., Ramírez A.C., Croche R., Baltazar-Zamora M.A. (2019). "Influencia del medio ambiente de exposición y tipo de curado en la cinética de corrosión de concreto reforzado" Memorias del XXXIV Congreso Nacional de la Sociedad Mexicana de Electroquímica, 12th Meeting of the Mexican Section of the Electrochemical Society, SMEQ, Querétaro (México).

García D.L, Cabral J.A, Gaona C., Almeraya F., Ramírez A.C., Villegas R., Marquez S., BaltazarZamora M.A. (2019). "Eficiencia anticorrosiva del acero AISI 304 como refuerzo en concretos ecológicos ternarios base CBCA-HS expuestos a sulfato de magnesio". Memorias del XXXIV Congreso Nacional de la Sociedad Mexicana de Electroquímica, 12th Meeting of the Mexican Section of the Electrochemical Society, SMEQ, Querétaro (México).

Jagadesh, P., Ramachandramurthy, A., Murugesan, R., \& Sarayu, K. (2015). "Micro-analytical studies on sugar cane bagasse ash". Indian Academy of Sciences , 1629-1638.

Jofre, C., Kraemer, C., Sampedro, A., Lopez Bachiller, A., Atienza, M., Diaz, M. (2008). Manual de estabilización de suelos con cemento o cal. Madrid: Instituto Español del cemento y sus aplicaciones. Juarez Gutierrez, O., Inzunza Ortiz, M. A. (2011). Guía practica de estabilización y recuperación de pavimentos con cemento portland en México. Ciudad de México: AMIVTAC.

Landa A., Gaona C., Almeraya F., Ramírez A.C., López O. M., Vélez J.A., Solorzano R., Márquez S., Baltazar-Zamora M.A., (2019). "Evaluación electroquímica del acero inoxidable AISI 316 embebido 
en concretos sustentables base ceniza de bagazo de caña de azúcar expuestos a un medio marino" Memorias del XXXIV Congreso Nacional de la Sociedad Mexicana de Electroquímica, 12th Meeting of the Mexican Section of the Electrochemical Society, SMEQ, Querétaro (México).

Landa A., Landa A., Rangel J.M., Almeraya F., Croche B., Baltazar-Zamora M.A. (2019). "Comportamiento de la corrosión del acero AISI 316 como refuerzo en concretos ternarios sustentables con sustitución parcial de CPC en 10\%, 20\% y 30\% de CBCA-HS inmersos en agua de mar". Memorias del XXXIV Congreso Nacional de la Sociedad Mexicana de Electroquímica, 12th Meeting of the Mexican Section of the Electrochemical Society, SMEQ, Querétaro (México).

Landa A.E., Croche R., López O.M., Galván R., Cabral J.A., Gaona C., Almeraya F., Baltazar-Zamora M.A (2018). Corrosion Behavior of AISI 316 Stainless Steel As Reinforcement in Ternary Sustainable Concrete Based on SCBA-SF Exposed in Seawater, ECS Meeting Abstracts, MA2018-02 584-584

Landa A.E., Castillo S., Reyes J.A., Villegas R., Galván R., Cabral J.A., Gaona C., Almeraya F., Baltazar-Zamora M.A. (2018). Evaluation of the Corrosion of AISI 304 Stainless Steel Embedded in Sustainable Concrete with High Volumes of Scba-SF Exposed in Marine Environment, ECS Meeting abstracts, MA2018-02 587

Landa A.E., Croche R., Márquez S., Villegas R., Ariza H.A., Estupiñan F., Gaona C., Almeraya F., Baltazar-Zamora M.A (2018). Corrosion Behavior 304 and 316 Stainless Steel as Reinforcement in Sustainable Concrete Based on Sugar Cane Bagasse Ash Exposed to $\mathrm{Na}_{2} \mathrm{SO}_{4}$, ECS transaction, 84, 179188

Landa A.E., Croche R., Márquez S., Galván R., Gaona C., Almeraya F., Baltazar-Zamora M.A. (2018). Correlation of Compression Resistance and Rupture Module of a Concrete of Ratio w/c=0.50 with the Corrosion Potential, Electrical Resistivity and Ultrasonic Pulse Speed, ECS, 84, 217-227

Moraes, J. C., Akasaki, J. L., Melges, J. L., Monzo, J., Borrachero, M. V., Soriano, L., et al. (2015). "Assessment of sugar cane straw ash (SCSA) as pozzolanic material in blended portland cement: microstructural characterization of pastes and mechanical strength of mortars". Construction and Building Materials, 670-677.0

Organismo Nacional de Normalización y Certificación de la Construcción y Edificación, S. C. (2014) "NMX-C-414-ONNCCE-2014: Industria de la Construcción-Cementantes HidráulicosEspecificaciones y Métodos de Ensayo", México, ONNCCE, S.C.

Ruíz M., Landa A., Cabral J.A., Gaona C., Almeraya F., Malpica R., Márquez S., Baltazar M.A. (2019) "Influencia del tipo de acero en el comportamiento de la corrosión de concretos sustentables ternarios elaborados con ceniza de bagazo de caña de azúcar y humo de sílice expuestos a sulfato de sodio" Memorias del XXXIV Congreso Nacional de la Sociedad Mexicana de Electroquímica, 12th Meeting of the Mexican Section of the Electrochemical Society, SMEQ, Querétaro (México).

Santiago, G., Baltazar-Zamora M.A., Galván, R., López, L.D., Zapata, F., Zambrano, P., A. Gaona, C. and Almeraya, F. (2016), Electrochemical evaluation of reinforcement concrete exposed to soil type sp contaminated with sulphates. Int. J. Electrochem. Sci. 11,4850-4864, doi: https://doi.org/10.20964/2016.06.31

Santiago, G., Baltazar-Zamora M.A., Olguín, J., López, L.D., Galván, R., Ríos, A. Gaona, C. and Almeraya, F. (2016), "Electrochemical evaluation of a stainless steel as reinforcement in sustainable concrete exposed to chlorides". Int. J. Electrochem. Sci. 11, 2994

Singh N.B., Singh V.D., Rai S. (2000), "Hydration of bagasse ash-blended portland cement". In Cement and Concrete Research. 30(9):1485-1488. https://doi.org/10.1016/S0008-8846(00)00324-0

Torres Rivas, B. J., Gaitan Arevalo, J. R., Espinoza Perez, L. J., \& Escalante Garcia, J. I. (2014). "Valoración de ceniza de bagazo de caña de la industria azucarera Nicaragüense como sustituto parcial al cemento portland" . Nexo Revista Científica , 82-89.

Volpi, V., López L.D., Hernández, J., Baltazar, M.A., Olguín, F.J. and López, A.L. (2017), “Corrosion study in reinforced concrete made with mine waste as mineral additive”. Int. J. Electrochem. Sci. 12,2231, doi: https://doi.org/10.20964/2017.01.08. 